ESAIM: PROCEEDINGS, October 2007, Vol. 22, 102-107

Gabriel Caloz \& Monique Dauge, Editors

\title{
PERFORMANCE EVALUATION OF A MULTISTRUCTURE METHOD OF MOMENTS
}

\author{
D. Arnaud-Cormos ${ }^{1}$, R. LoIson $^{1}$ And R. Gillard ${ }^{1}$
}

\begin{abstract}
In this paper, a MultiStructure computation technique based on the Method of Moments (MSMoM) is presented. The technique permits the simultaneous analysis of different structures, such as printed antennas, with only one electromagnetic (EM) simulation. Its performance in terms of number of operations for the analysis of several structures is evaluated. The complexity of the new technique is considerably reduced by comparison to an equivalent direct MoM implementation leading to important time savings.
\end{abstract}

Résumé. Dans cet article, une technique de calcul MultiStructure basée sur la Méthode des Moments est présentée. Cette technique permet l'analyse simultanée de plusieurs structures, comme par exemple les antennes imprimées, avec une seule simulation électromagnétique. Ses performances en terme de nombre d'opérations pour l'analyse de plusieurs structures sont évaluées. Il en ressort que la nouvelle technique est beaucoup moins gourmande en nombre d'opérations que la MoM classique permettant ainsi de réduire le temps le calcul.

\section{INTRODUCTION}

Printed antennas are structures made of dielectric layers which support metallic parts of any shape. They are fabricated using printed circuits technologies and they are low cost and compact. Therefore, they are used in many communication systems such as mobile technology, wireless systems (WIFI, Bluetooth) or spatial communications. Printed antennas are also used for radar or RFID tags applications for frequencies going from $1 \mathrm{GHz}$ to millimeter wave band. Antennas are designed by CAD software tools which are based on the resolution of Maxwell equations. Among existing numerical techniques for the EM modeling of the antennas, the most used are the finite element method, the method of moments for resolution by integral equations or the finite difference time domain method.

In this paper, a new approach based on the method of moments [1] is presented. Although accurate and efficient for small-scale electromagnetic problems, the classical MoM can be very time consuming with the increase of the electrical size of circuits. Simulation time can also become prohibitive in an optimization process where several iterations are performed [2]. The new approach called "multistructure" was developed in order to speed up classical MoM. The original idea of MSMoM is to simultaneously analyze several structures whose metallic parts are slightly different, using a single simulation. This concept avoids a large amount of re-computation, and therefore, permits drastic reduction of the computation time when tuning a structure to be optimized.

\footnotetext{
${ }^{1}$ Institut d'Electronique et de Télécommunications de Rennes (IETR), 20 avenue des buttes de Coësmes, CS 14315, 35043 Rennes cedex, France.
}

(C) EDP Sciences, SMAI 2007 
The objective of the present paper consists in evaluating the MSMoM complexity by comparison with the classical MoM complexity. This paper is organized as follows. The general principles of the classical MoM and the new MSMoM are presented in Section 1. In Section 2, the MSMoM complexity is compared to that of a classical MoM. Finally, Section 3 draws some conclusions.

\section{THEORY}

\subsection{MoM Formulation}

The analyzed structures are printed structures. Figure 1 shows a typical analyzed structure.

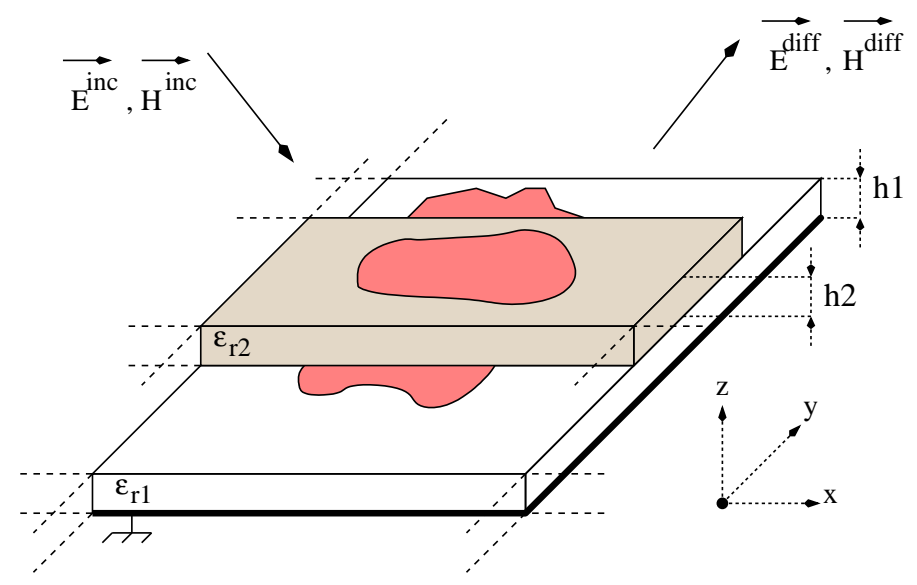

Figure 1. Typical analyzed structure.

It consists of metallizations located at different interfaces of stacked dielectric layers. Electromagnetic problems involving conductors (for instance, printed structures) can be formulated using an electric-field integral equation (EFIE). This equation relates the electric currents induced on the metallic surface to the incident electric field. Classically, the EFIE expresses the cancellation of the total tangential electric field at the surface of the perfect electric conductors:

$\vec{e}_{n}\left(\vec{r}_{\text {obs }}\right) \times \vec{E}^{i n c}\left(\vec{r}_{\text {obs }}\right)=\vec{e}_{n}\left(\vec{r}_{\text {obs }}\right) \times\left\{\iint_{S_{\text {sou }}}\left[j \omega \overline{\bar{G}}_{A}\left(\vec{r}_{\text {obs }} / \vec{r}_{\text {sou }}\right) \cdot \vec{J}_{s}\left(\vec{r}_{\text {sou }}\right)+\vec{\nabla}_{\text {obs }} G_{V}\left(\vec{r}_{\text {obs }} / \vec{r}_{\text {sou }}\right) \rho_{s}\left(\vec{r}_{\text {sou }}\right)\right] d S_{\text {sou }}\right\}$,

In this equation, $\vec{E}^{i n c}$ is the incident electric field; $\vec{r}_{o b s}$ locates an observing point at the surface of the metallization; $\omega$ is the pulsation; $\bar{G}_{A}$ and $G_{V}$ are the dyadic and scalar dielectric multi-layer Green's functions; $\vec{r}_{\text {sou }}$ locates a source point at the surface of a metallization; $\vec{J}_{s}$ and $\rho_{s}$ are the current and charge densities at the source point. As $\vec{J}_{s}$ and $\rho_{s}$ are related by the continuity equation, the unknown of the integral equation is the current density flowing on the conductors of the studied structure.

Introduced to the electromagnetic community by Harrington [1], the method of moments is among the most used numerical techniques to transform the integral equation (1) into a finite linear system. The first step of the method consists in discretizing the unknown on the metallic surface using elementary cells. Then, the current density is expanded on a set of $N$ basis functions defined on the metallizations. The development of the current density leads to a discretized integral equation with $N$ unknowns. The second step of the numerical technique consists in projecting the discretized integral equation on $N$ weighting functions defined on the metallizations. 
When the basis functions and the weighting functions are identical, the MoM is more known as the Galerkin method. The weighting step leads to a complex linear system of dimension $N$ defined as follows:

$$
Z I=V,
$$

where $Z$ is the generalized impedance matrix representing all electromagnetic interactions, $V$ stands for the excitation vector accounting for the incident field and $I$ is the vector of the unknown currents induced on the metallic surface. Once the linear system is solved by direct or iterative methods, the current distribution is known in a discrete form. It is then possible to determine the electromagnetic characteristics such as the input impedance or the radiation patterns of the studied printed structure. The computation of the currents is the most time-consuming operation. To accelerate this operation, an original technique presented in the next section has been developed.

\subsection{MSMoM Formulation}

\subsubsection{Principle of the method}

In the MSMoM approach, a mother structure (MS) with a large metallic part discretized in elementary cells is first considered (figure 2). It defines a linear system whose resolution yields the electric current densities flowing on the MS.

MS

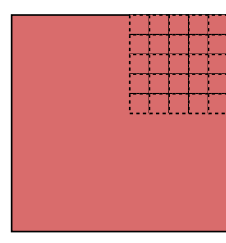

suppressed cell

$\mathrm{DS}^{1}$
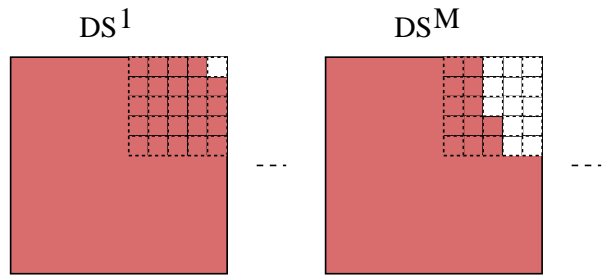

Figure 2. Extraction of daughter structures (DS) from a mother structure (MS).

Daughter structures (DS) are extracted from the MS by suppressing elementary cells, i.e., by eliminating the currents on these cells. As demonstrated in [3], this can be achieved without solving any new linear system. Indeed, all the information required to study the DS is already present in the MS analysis. In practice, currents on any DS are deduced rigorously from the original currents flowing on the MS using a simple analytical postprocessing. This post-processing only requires additional excitation vectors for each expected DS in the original MS linear system. This technique can also be described by viewing each cell as a port. A short circuited port corresponds to a metallized cell while an open port is equivalent to a suppressed cell. In the next section, the development of the method is presented. More precisely, the approach is detailed for the case when only one cell is removed.

\subsubsection{Development of the method}

For the MSMoM development, the MS is called structure 0, the first DS (one removed cell) is called structure 1, the second DS (two removed cells) is called structure 2 and so on. Then, for $M$ different DSs, the original system to be solved is given by 


$$
Z^{0} \cdot\left[I^{00}, I^{01}, \ldots, I^{0 M}\right]=\left[V^{0}, V^{1}, \ldots, V^{M}\right]
$$

where $V^{0}$ is the actual excitation vector (the same for all structures), $V^{n}, n>0$, is an excitation vector with zero elements except the element corresponding to the current to be eliminated, $I^{m n}$ is the current vector on structure $m$ in response to the excitation vector $V^{n}$.

Many different DS can be obtained from a given mother, each of them involving a different number of removed cells. For simplicity, we consider the case of a single DS with only one suppressed cell (we note it cell $j$ ). It may, for example, corresponds to $D S^{1}$ in figure 2. We define $I^{10}$ as the current on $D S^{1}$ and $I^{00}$ as the current on the MS. To obtain $D S^{1}$ solution, we first note that the DS can be thought as the original MS with port $j$ loaded by an open circuit (this particular loading configuration permits to cancel current $I_{j}$ as required for the DS). This means that the matrix equation representing the DS can be written as:

$$
\left(Z+Z_{L}\right) I^{10}=V,
$$

where $Z_{L}$ is a matrix with null elements except the diagonal term j:

$$
\begin{gathered}
\left(Z_{L}\right)_{k l}=Z_{O C} \quad \text { for } \quad k=l=j, \\
\left(Z_{L}\right)_{k l}=0 \quad \text { elsewhere }
\end{gathered}
$$

Then, multiplying (4) by $Y=Z^{-1}$, reminding that $I^{00}=Y V$ and making $Z_{O C}$ infinite, we obtain:

$$
\begin{aligned}
I_{j}^{10} & =0 \\
I_{k}^{10} & =I_{k}^{00}-\frac{I_{k}^{01}}{I_{j}^{01}} I_{j}^{00}, \quad \forall k, k \neq j .
\end{aligned}
$$

As a result, equation (7) permits to obtain the current on the DS from a simple manipulation of the current on the MS. Note that both current vectors $I^{01}$ and $I^{00}$ are available since they were calculated with the original system (3).

For the general case, when $M$ different DSs are involved, a recursive approach is performed as described in [3]. The complexity of the general process is less important than for several individual simulations (for the MS and for all the DS) as will be shown in the next section.

\section{MSMOM EFFICIENCY EVALUATION}

In this section, the computational complexity of the new technique is compared to the one of a conventional MoM. The total number of operations required for the analysis of several structures (a MS and $M$ DSs) is calculated for both methods. The linear system obtained either for the MoM or the MSMoM is solved by a direct Gaussian elimination method.

\subsection{MoM complexity}

The number of operations required by the classical MoM to solve the linear system (2) is given by:

$$
\operatorname{Nbop}^{\operatorname{MoM}}(N)=\left[\frac{N^{3}}{3}+\frac{3 N^{2}}{2}-\frac{11 N}{6}\right]+\left[\frac{N^{3}}{3}+\frac{3 N^{2}}{2}-\frac{11 N}{6}\right]+\left[\frac{N^{2}}{2}+\frac{N}{2}\right]
$$

where $N$ is the order of the linear system, that is to say the number of unknowns of the problem.

The first term corresponds to the number of additions, the second is the number of multiplications and the last one represents the number of divisions. 
The number of operations required by the classical MoM for the simulation of a MS and $M$ DSs is expressed by:

$$
\operatorname{Nbop}^{\text {Total-MoM }}(M, N)=\sum_{i=0}^{M} \operatorname{Nbop}^{\mathrm{MoM}}(N-i)
$$

where $i$ is the number of deleted currents from the MS. Remember that each deleted current corresponds to a new DS.

\subsection{MSMoM complexity}

The MSMoM complexity is obtained by adding two quantities.

The first one is the number of operations required by the MSMoM to solve the linear system (3) of order $N$ with $P=M+1$ additional vectors:

$$
\operatorname{Nbop}^{\mathrm{SM}}(P, N)=\left[\frac{N^{3}}{3}+\frac{3 P N^{2}}{2}-\left(\frac{2 N+9 P N}{6}\right)\right]+\left[\frac{N^{3}}{3}+\frac{3 P N^{2}}{2}-\left(\frac{2 N+9 P N}{6}\right)\right]+\left[\frac{N^{2}}{2}+P N-\frac{N}{2}\right]
$$

The second quantity is the number of operations required by the MSMoM to delete $M$ currents:

$$
\operatorname{Nbop}^{\text {Delete }}(M, N)=\left[\frac{M^{2} N}{2}+\frac{M N}{2}\right]+\left[\frac{M^{2} N}{2}+\frac{M N}{2}\right]+\left[\frac{M^{2} N}{2}+\frac{3 M N}{2}\right]
$$

So, the total number of operations required by the MSMoM for the simulation of a MS and $M$ DSs is expressed by:

$$
\operatorname{Nbop}^{\text {Total-MSMoM }}(M, N)=\operatorname{Nbop}^{\mathrm{SM}}(M+1, N)+\operatorname{Nbop}^{\text {Delete }}(M, N)
$$

\subsection{Comparison of the complexity for the MoM and the MSMoM}

The number of operations required by the MoM and the MSMoM are calculated for several linear system orders : $N=10,25,1000$ and 10000. These systems correspond to 4 different MS. For each case, the number of operations used to obtain $M$ DSs is also calculated. The number $M$ varies between $0 \%$ and $100 \%$ of $N$ with a step of $10 \%$. The complexities of the MoM (equation (9)) and of the MSMoM (equation (11)) are compared in figures 3 and 4 (logarithmic scale).

As shown in figure 3(a), for small system orders, the MoM requires less operations than the MSMoM. But for system orders larger than 25, the MSMoM becomes competitive compared to the conventional MoM (figure $3(\mathrm{~b})$ ). Actually, the larger is the system order, the more competitive is the MSMoM in comparison with the MoM (figure 4). Moreover, the MSMoM is particularly adapted for an optimization process in which an initial structure is progressively updated by small variations around a given geometry.

\section{Conclusion}

In this paper, the general principles of a MultiStructure technique which permits to speed up the MoM have been presented. The technique permits the simultaneous and rigorous analysis of different structures with only one electromagnetic simulation. The complexity of the new MSMoM technique has been calculated and compared to the complexity of a classical MoM. It shows that the MSMoM is very competitive as the linear system order increases.

Moreover, the MSMoM is particularly suitable for an iterative optimization procedure. As shown in [4], when combined with a genetic algorithm, it permits a significant reduction of the computational time required to optimize complex geometries. 


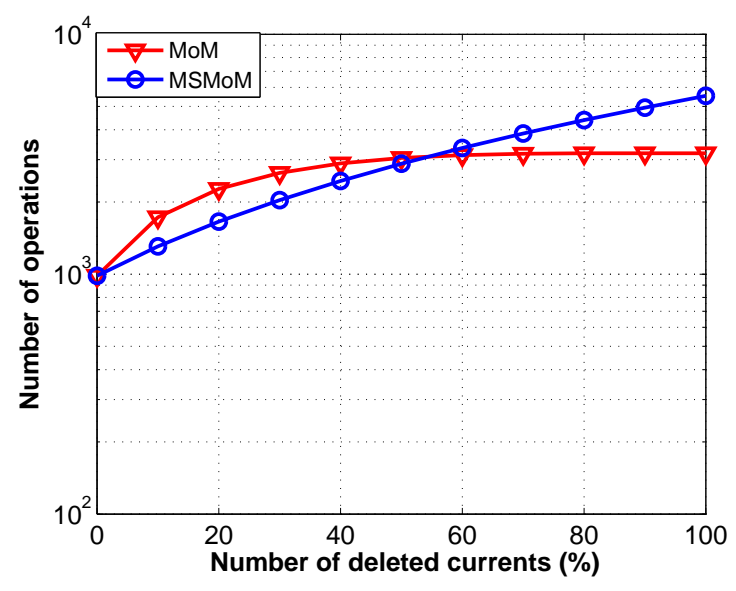

(a) $\mathrm{N}=10$

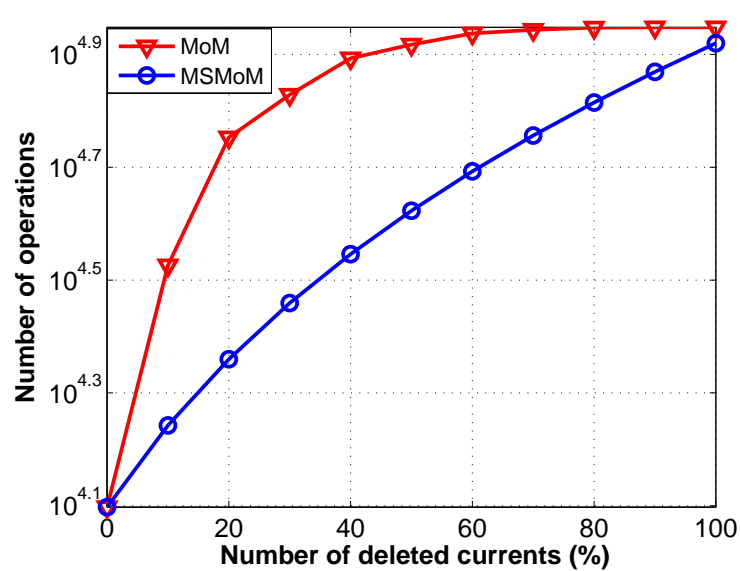

(b) $\mathrm{N}=25$

Figure 3. MoM and MSMoM complexities for $N=10$ and 25.

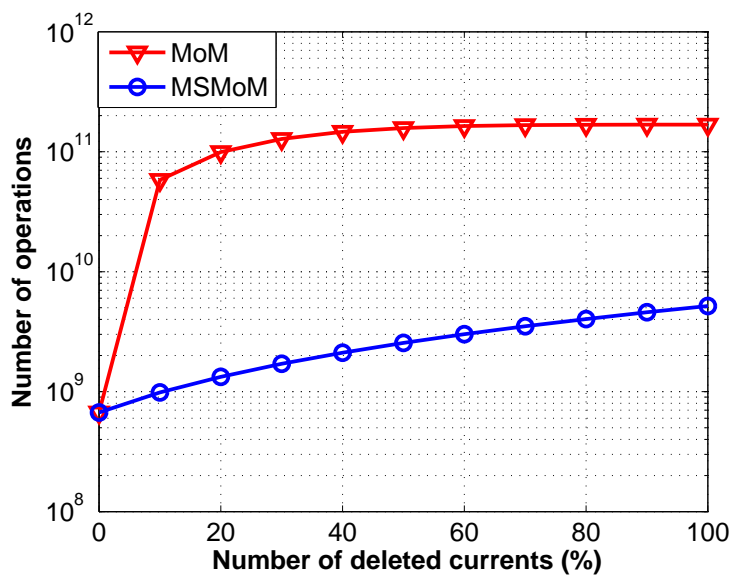

(a) $\mathrm{N}=1000$

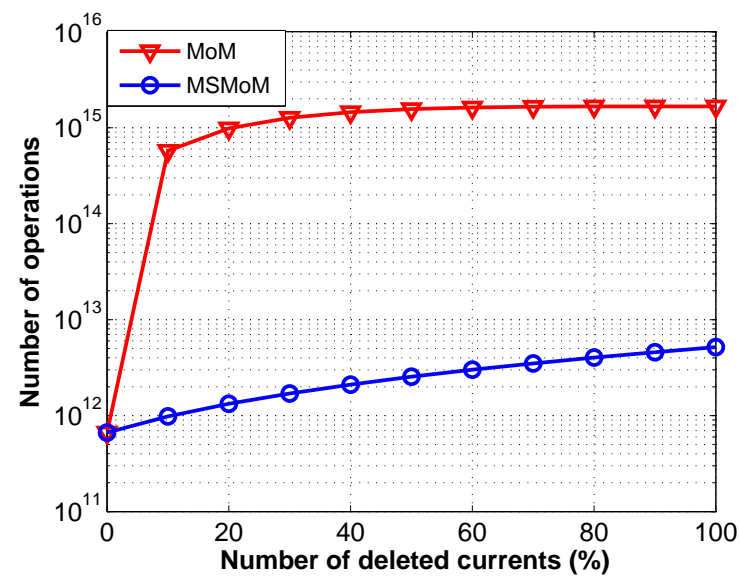

(b) $\mathrm{N}=10000$

Figure 4. MoM and MSMoM complexities for $N=1000$ and 10000.

\section{REFERENCES}

[1] R. F. Harrington, Field Computation by Moment Method, Macmillan Series in Electrical Science, New York, 1968.

[2] J. M. Johnson and Y. Rahmat-Samii, "Electromagnetic Optimization by Genetic Algorithms", Wiley Series in Microwave and Optical Engineering, John Wiley \& sons, inc, New York 1999, pp. 249-278.

[3] D. Cormos, R. Loison, and R. Gillard, "A Multistructure Method of Moment for EM Optimization", Microwave Opt. Technol. Lett., Vol. 40, N2, pp. 114-117, Jan. 2004.

[4] D. Cormos, R. Loison et R. Gillard, "Fast Optimization and Sensitivity Analysis of Nonintuitive Planar Structures", IEEE Microwave Theory and Techniques, Vol. 53, N 6, pp. 2019-2025, Juin 2005. 